University of Wollongong

Research Online

Faculty of Social Sciences - Papers (Archive) Faculty of Arts, Social Sciences \& Humanities

$1-1-2016$

\title{
Pulse modulated radiofrequency exposure influences cognitive performance
}

Adam Verrender

University of Wollongong, av138@uowmail.edu.au

Sarah P. Loughran

University of Wollongong, loughran@uow.edu.au

Anna Dalecki

University of Wollongong, adalecki@uow.edu.au

Raymond J. McKenzie

Swinburne University of Technology, Ray.McKenzie@team.telstra.com

Rodney J. Croft

University of Wollongong, rcroft@uow.edu.au

Follow this and additional works at: https://ro.uow.edu.au/sspapers

Part of the Education Commons, and the Social and Behavioral Sciences Commons

Research Online is the open access institutional repository for the University of Wollongong. For further information contact the UOW Library: research-pubs@uow.edu.au 


\title{
Pulse modulated radiofrequency exposure influences cognitive performance
}

\author{
Abstract \\ Purpose: To investigate whether exposure to pulse modulated radiofrequency (PM RF) influences human \\ cognitive performance, and whether it does so in a dose-dependent manner. Materials and methods: \\ Thirty-six healthy adults participated in a randomized, double-blind, counterbalanced provocation study. \\ Cognitive performance was assessed using a visual discrimination task and a modified Sternberg \\ working memory task, which were calibrated to individual performance levels in a preliminary testing \\ session. An sXh920 planar exposure system was used to generate a $920 \mathrm{MHz}$ GSM-like signal, providing \\ three conditions (peak-spatial SAR averaged over $10 \mathrm{~g}$ ) of $0 \mathrm{~W} / \mathrm{kg}$ (sham), $1 \mathrm{~W} / \mathrm{kg}$ (low RF) and $2 \mathrm{~W} / \mathrm{kg}$ \\ (high RF). Results: A significant decrease in reaction time (RT) in the Sternberg working memory task was \\ found during exposure compared to sham. This effect was not dose-dependent. Conclusions: Cognitive \\ performance was shown to be faster under PM RF conditions, relative to sham, in a working memory \\ task. While the majority of the literature has not found effects of PM RF exposure on cognitive \\ performance, it is possible that the methodological improvements employed in the present study \\ increased sensitivity, and thus the ability to detect potential effects. \\ Keywords \\ radiofrequency, exposure, modulated, influences, cognitive, performance, pulse \\ Disciplines \\ Education | Social and Behavioral Sciences \\ Publication Details \\ Verrender, A., Loughran, S. P., Dalecki, A., McKenzie, R. J. \& Croft, R. J. (2016). Pulse modulated \\ radiofrequency exposure influences cognitive performance. International Journal of Radiation Biology, 92 \\ (10), 603-610.
}


Title Page

\section{Pulse modulated radiofrequency exposure influences cognitive performance}

Adam Verrender ${ }^{1,2}$

Sarah P. Loughran ${ }^{1,2,3}$

Anna Dalecki ${ }^{2,3}$

Ray McKenzie ${ }^{1,4}$

Rodney J. Croft ${ }^{1,2,3}$

${ }^{1}$ Australian Centre for Electromagnetic Bioeffects Research

${ }^{2}$ Centre for Health Initiatives, School of Psychology, Illawarra Health \& Medical Research Institute, University of Wollongong, Australia

${ }^{3}$ Population Health Research on Electromagnetic Energy, Australia

${ }^{4}$ Australian Mobile Telecommunications Association

Address for correspondence: Adam Verrender. Australian Centre for Electromagnetic Bioeffects Research, Illawarra Health and Medical Research Institute, Bld 32.113, University of Wollongong, Northfields Ave, Wollongong, NSW, 2522, Australia

Tel: +612 4239 2118; Fax: +612 4221 8130; Email: av138@uowmail.edu.au

Keywords: Mobile Phones, Cognition, Working Memory, RF Bioeffects

Running Title: PM RF influences cognitive performance 


\begin{abstract}
Purpose: To investigate whether exposure to pulse modulated radiofrequency (PM RF) influences human cognitive performance, and whether it does so in a dose dependent manner.
\end{abstract}

Materials and Methods: Thirty six healthy adults participated in a randomised, double blind, counterbalanced provocation study. Cognitive performance was assessed using a visual discrimination task and a modified Sternberg working memory task, which were calibrated to individual performance levels in a preliminary testing session. An sXh920 planar exposure system was used to generate a $920 \mathrm{MHz}$ GSM-like signal, providing three conditions (peakspatial SAR averaged over 10g) of $0 \mathrm{~W} / \mathrm{kg}$ (Sham), $1 \mathrm{~W} / \mathrm{kg}$ (Low RF) and $2 \mathrm{~W} / \mathrm{kg}$ (High RF).

Results: A significant decrease in reaction time (RT) in the Sternberg working memory task was found during exposure compared to Sham. This effect was not dose dependent.

Conclusions: PM RF exposure was shown to influence cognitive performance in a working memory task. While the majority of the literature has not found effects of PM RF exposure on cognitive performance, it is possible that the methodological improvements employed in the present study increased sensitivity, and thus the ability to detect potential effects. 


\section{Introduction}

Over the past two decades, the increasingly widespread use of mobile phones has generated growing concern about potential adverse effects that the radiofrequency electromagnetic fields (RF-EMF) emitted by these devices could have on human health. While a number of independent reviews have concluded that there are no substantiated health effects associated with exposure to mobile phone RF-EMF (World Health Organisation, 2014, Health Canada, 2015, SCENIHR, 2009, Health Council of the Netherlands, 2009), there is evidence which indicates that exposure can influence the brain's electrical activity. Specifically, it has been consistently shown that pulse modulated RF (PM RF) affects spontaneous resting alpha (8-12 Hz) (Croft et al., 2008, Croft et al., 2010, Leung et al., 2011, Perentos et al., 2007, Regel et al., 2007a, Curcio et al., 2005) and sleep spindle activity (approximately 11-15 Hz) in nonrapid eye movement sleep (Huber et al., 2002, Regel et al., 2007b, Schmid et al., 2012, Loughran et al., 2005, Huber et al., 2000).

However, the functional consequence of this change in EEG activity remains unclear, as studies assessing aspects of cognitive and behavioural functioning during and following exposure to PM RF have produced contradictory, but mostly null results (Barth et al., 2012, Valentini et al., 2010).

One measure of cognitive performance which has yielded inconsistent results is working memory performance, which has been primarily assessed using the N-back task. Three studies have reported decreases in reaction time (Koivisto et al., 2000, Regel et al., 2007a, Regel et al., 2007b) and one study reported an improvement in accuracy (Regel et al., 2007b). The majority of studies using this task, however, have not found any effects of PM RF exposure on performance (Haarala et al., 2003, Haarala et al., 2004, Haarala et al., 2007, Krause et al., 2007, Leung et al., 2011). 
The difficulty in establishing a firm conclusion as to whether PM RF influences cognitive performance may be attributed to a number of methodological constraints. For example, the variation in methods between research groups makes it difficult to compare or verify previous results, while poor exposure protocols and experimental designs, inadequate sample sizes and a lack of reliable cognitive performance measures have limited the potential for finding an effect (Regel and Achermann, 2011). Several other issues may have also contributed to the mixed findings. For instance individual differences in cognitive performance have not been adequately accounted for in previous studies. It is also possible that the N-back task is not sensitive enough to adequately detect changes in cognitive performance as a result of PM RF exposure (Regel and Achermann, 2011) as it has been shown that this task can be significantly influenced by learning effects (Regel et al., 2007a, Regel et al., 2007b, Haarala et al., 2004, Haarala et al., 2005).,. Furthermore, as the only known interaction between RF exposure and the body is via heating (Adair and Black, 2003), thermally induced variability may have also influenced the results. In addition, the localised, intermittent exposure protocols utilised by some research groups may not have been sufficiently powerful to produce an effect (Boutry et al., 2008). These factors have the potential to introduce large amounts of error variance, which may have masked any potential real effects.

In order to overcome these issues, the present study has been designed with several methodological improvements to examine whether exposure to PM RF influences cognitive performance in a dose dependent manner. In particular, a visual discrimination task and a modified Sternberg working memory task (Sternberg, 1966) were employed, both of which were calibrated to individual performance levels in a preliminary testing session. The study also utilised a planar patch antenna system to ensure that there was a consistent, uniform exposure across the target hemisphere. Thermally induced variability was reduced by clamping skin temperature to a thermo-neutral level. In addition to these improvements, the 
cognitive performance data was treated using an index of the participants' response sensitivity and bias, as adapted from signal detection theory (Stanislaw and Todorov, 1999). This treatment takes into account how well a participant can discriminate between trials (sensitivity) and the participant's general tendency to respond with a 'yes' or 'no' button press (bias), giving a better indication of task accuracy and minimising Type I and Type II error. Further to the double-blind, counterbalanced, Sham controlled design, these improvements were implemented to increase sensitivity, and thus, the possibility of finding potential effects. The results of the present study are part of a larger study investigating the effect of PM RF exposure on the electroencephalogram and thermoregulatory processes. 


\section{Materials and Methods}

\section{Participants}

Forty three participants were recruited through advertisements and word of mouth. Seven participants failed to attend all testing sessions, leaving thirty six participants (half male) aged $18-52$ years $(\mathrm{M}=24.44 ; \mathrm{SD}=6.27)$. To be included in the study, participants were required to be between 18 and 55 years of age, be right handed and report being of good health. Participants were excluded from the study if they reported having a current illness or medical condition, or having used illicit substances within the 7 day period prior to the study. Suitable participants were required to attend the Illawarra Health and Medical Research Institute, University of Wollongong, for four mutually convenient testing sessions, at the same time of day and separated by a period of at least 7 days. The study was approved by the Human Research Ethics Committee (University of Wollongong: HE13/146), and written, informed consent was obtained from all participants. All participants were instructed to abstain from alcohol for at least 8 hours before the commencement of a testing session, abstain from caffeine for at least 1 hour before a testing session, and to not use their mobile phone for at least 2 hours before the beginning of a testing session. All participants were compensated a total of $\$ 200$ for their involvement in the study.

\section{Radiofrequency exposure}

An sXh920 planar exposure system (IT'IS Foundation, Zurich, Switzerland) was used to generate a $920 \mathrm{MHz}$ GSM-like signal (as emitted by a mobile phone handset in active mode while transmitting voice). The signal included the basic GSM frequency components $(8.33,216.6,1733 \mathrm{~Hz}$, including corresponding harmonics; crest factor $=8.3)$. Two RF antennas placed on wooden pillars were positioned $42 \mathrm{~mm}$ vertically above the ear canal at a distance of $115 \mathrm{~mm}$ from the head (Huber et al., 2002, Huber et al., 2005, Loughran et al., 2013). The RF exposure of the sXh920 system has been fully characterised and was 
calibrated to provide a peak-spatial SAR averaged over $10 \mathrm{~g}$ of $0 \mathrm{~W} / \mathrm{kg}, 1 \mathrm{~W} / \mathrm{kg}$ and $2 \mathrm{~W} / \mathrm{kg}$, for the Sham, Low and High exposure conditions respectively (for full dosimetric data see Murbach et al. (2012)). These exposures were within the Australian general public RF exposure limits (ARPANSA RPS3). The system was controlled electronically using defined participant numbers. The fully randomised and counterbalanced exposure conditions were assigned to each participant and preprogrammed by a researcher not involved in the collection of data (RC) to ensure that double blinding was maintained. An inbuilt failsafe mechanism ensured RF levels did not exceed RPS3 levels. Only the left hemisphere was exposed to RF, and brown noise was used to mask any sounds made by the exposure system in order to ensure that participants were not aware of the exposure condition. At the completion of each experimental testing session, participants were asked whether they were aware of the exposure status and the side of exposure via a pen and paper Likert scale $(1=$ 'left', 2 = 'right', 3 = 'both', 4 = 'no', 5 = 'don't know') and an open ended question asking for further details about their ability to perceive the exposure ('If yes, how did you perceive the field?').

\section{Design}

A double blind, counterbalanced, cross-over design was employed. Following a preliminary calibration session, each participant's cognitive performance was tested under three conditions (Sham, Low and High RF) during exposure (block 1) and following exposure (block 2) over three separate sessions separated by at least seven days. RF exposure was emitted to the left hemisphere only, and participants were not made aware of which antenna was emitting. Order of exposure was counterbalanced across participants and randomly assigned. 


\section{Cognitive Performance Tasks}

Two tasks were used to assess cognitive performance. These tasks were calibrated to each participant's individual level of performance in a preliminary testing session and sufficiently long practice blocks were utilised prior to each experimental session to reduce learning, floor and ceiling effects. Behavioural outcomes were measured as the number of correct hits, correct rejections, false alarms and false rejections, as well as participant's reaction time (RT) to correct responses.

Visual discrimination task. Visual perception was assessed using a visual discrimination task. A series of crossed white lines were presented in the centre of a computer monitor with a black background for $200 \mathrm{~ms}$ each. Between each stimulus presentation, a mask appeared for $500 \mathrm{~ms}$, followed by a blank screen for $300 \mathrm{~ms}$. Figure 1 presents an example of the stimuli and mask used in the visual discrimination task.

\section{[Insert Figure 1 here]}

For each stimulus presentation, participants were asked to respond as quickly and accurately as possible via a 'yes' button press with the right thumb if they thought that the lines differed in thickness (target stimulus), or not to respond if they thought that the lines did not differ in thickness (non-target stimulus). A total of 180 stimuli were presented (half targets). The frequency and presentation of the target stimuli were pseudo-randomised across trials and balanced between blocks.

Task difficulty was manipulated by increasing the magnitude of thickness between the two lines in target stimuli. The greater the difference between the two lines, the easier the trial. In target stimuli, one line was kept at a constant thickness of $1.5 \mathrm{~mm}$ while the second line was manipulated in increments of $0.05 \mathrm{~mm}$. In non-target stimuli, both lines were kept constant at $1.5 \mathrm{~mm}$. 
Sternberg working memory task. Working memory performance was assessed using a modified Sternberg working memory task (Sternberg, 1966). The task involved memorising a stimulus set, maintaining that stimulus set in memory during a 3 second retention period, and recalling whether a subsequent probe stimulus was in the original memory set. The memory sets ranged in difficulty level from 6 to 15 letters.

The memory sets were constructed from a pool of 21 consonants (B, C, D, F, G, H, J, K, L, $\mathrm{M}, \mathrm{N}, \mathrm{P}, \mathrm{Q}, \mathrm{R}, \mathrm{S}, \mathrm{T}, \mathrm{V}, \mathrm{W}, \mathrm{X}, \mathrm{Y}$ and $\mathrm{Z}$ ) presented simultaneously in a horizontal arrangement over two lines. The letters were 66pt, capitalised, black and in bold Calibri font and presented in a centred $6.24 \times 25.40 \mathrm{~cm}$ grey box on a black background. Each letter was spaced $2 \mathrm{~cm}$ apart.

Figure 2 outlines the Sternberg working memory task design and progression for each trial. For each trial, participants were asked to respond as quickly and accurately as possible with a button press using the right thumb if the probe consonant appeared in the preceding memory set (target), or to not respond if the probe consonant was not presented in the memory set (non-target). Maximum response time was set at 2 seconds. 40 memory sets were presented (half targets). The frequency and presentation of the probe stimuli were pseudo-randomised across trials and balanced between blocks. The position of the target probe stimuli in the memory set was also pseudo-randomised across trials.

\section{[Insert Figure 2 here]}

Cognitive Task Calibration. For each participant, the cognitive tasks were calibrated during a preliminary testing session, to ensure that the tasks were at a level which was difficult, yet still achievable. In the visual discrimination task, the level of difficulty was defined as the two most difficult line manipulations that resulted in a response sensitivity score of 0.8 and 0.6, corresponding to the easy and difficult versions of the task respectively. In the Sternberg 
working memory task, this level was defined as the most difficult stimulus set that resulted in a response sensitivity value of 0.8 . To attain this value, participants completed four blocks. A five minute break separated each of the calibration blocks. In all blocks, the visual discrimination task preceded the Sternberg working memory task. During the calibration session, participants were not exposed to RF and no physiological data was recorded.

\section{EEG and thermo-physiological apparatus}

A water perfusion garment (Grant Instruments Ltd., Cambridge, U.K.) was used to clamp body skin temperature to $34^{\circ} \mathrm{C}$ to produce a thermo-neutral environment. The water perfusion suit was made of cotton and enclosed a series of pipes which distributed water across the skin at a rate of $2.5 \mathrm{~L} / \mathrm{min}$ at a temperature of $34{ }^{\circ} \mathrm{C}$. The garment covered the torso, arms, waist and legs. Water temperature was controlled using a digital thermostat (Type: GD120, Grant Instruments Ltd., Cambridge, U.K.) which could heat or cool water accordingly to $34{ }^{\circ} \mathrm{C} \pm .02^{\circ} \mathrm{C}$. A range of physiological measures, including core body temperature, skin temperature, blood pressure, resting EEG and cutaneous blood flow to the left hand were also acquired. These measures are beyond the scope of this paper and will not be discussed.

\section{Procedure}

Participants arrived at the laboratory at either 09:00 or 13:00, with the start time for all experimental sessions kept constant within-subjects to minimise circadian effects. Participants then completed a 16-item visual analogue mood scale (VAMS) and a series of short questionnaires asking about sleep, caffeine and alcohol consumption and mobile phone usage before being fitted with the water perfusion suit, EEG and physiological recording apparatus. 
Participants were then seated inside a Faraday cage in front of a Dell U2311H LCD monitor between the two RF antennas and the water perfusion suit was switched on. Participants were positioned such that their eyes were approximately $90 \mathrm{~cm}$ from and at the same height as the centre of the computer screen. The plane of the monitor was perpendicular to both the floor and the sagittal plane of the participants. Participants then completed a practice version of the visual discrimination and modified Sternberg working memory tasks (2.5 mins each). Once setup was complete, participants completed a 16 min 'Baseline' block, during which they were not exposed to RF. During this block participants completed an EOG correction task (Croft et al., 2005) and resting EEG and physiological data were also recorded.

The Baseline block was followed by two 30 min experimental blocks, the first block being 'RF ON' (Sham, Low or High RF depending on counterbalancing) and the second 'RF OFF'(post-exposure), with a 1 min break between each block. At the beginning of the experimental blocks, resting EEG and physiological measures were recorded. Subsequent to this, participants completed the cognitive battery; consisting of a 6 min visual discrimination task (easy and difficult consecutively) and a 9 min Sternberg working memory task, with a 1 min break between tasks. Following the cognitive tasks, EEG and physiological data were again recorded.

At the completion of testing, all monitoring equipment was disconnected and the participant completed a 16 item VAMS and an exposure condition questionnaire. This procedure was repeated for the remaining testing sessions.

\section{Data analyses}

Behavioural measures were defined as mean reaction time to correctly identified target probes ('RT') recorded in ms, as well as the number of correct hits, correct rejections, false alarms and false rejections $100-900 \mathrm{~ms}$ post stimulus in the visual discrimination task and 
100-2000 ms post probe stimulus in the Sternberg working memory task. Response sensitivity (Grier's A') and response bias (Grier's B") were used to assess task accuracy. All data points were converted to $z$ scores for analysis.

Where a participant performed at below $55 \%$ accuracy in the visual discrimination task or $50 \%$ accuracy in the Sternberg working memory task, data points were interpolated in order to preserve counterbalancing. This criterion affected 1 participant ( 3 blocks) in the difficult version of the visual discrimination task, and 6 participants (12 blocks) in the Sternberg working memory task.

Data points were missing for a further 2 participants ( 2 blocks for 1 participant, 1 block for 1 participant) in the visual discrimination task due to the incorrect difficulty level being administered. The missing data points were interpolated in order to preserve counterbalancing.

Statistical analyses were performed with SPSS statistical package 21.0. Paired samples $t$ tests were performed to assess the effect of exposure on RT, response sensitivity and response bias overall (comparing the $0 \mathrm{~W} / \mathrm{kg}$ condition against the average of the $1 \mathrm{~W} / \mathrm{kg}$ and $2 \mathrm{~W} / \mathrm{kg}$ conditions) and whether these effects depended on the dose of exposure (comparing the 1 $\mathrm{W} / \mathrm{kg}$ condition against the $2 \mathrm{~W} / \mathrm{kg}$ condition). Exploratory paired samples $t$ tests were also conducted on non-interpolated data. To correct normality, square root transformations were computed for the response sensitivity data in the easy version of the visual discrimination task. 


\section{Results}

\section{RF Status}

Overall, there was a greater tendency for participants to rate the exposure as being off $(50.93 \%)$ or unsure as to whether it was on or off $(37.05 \%)$ than on $(12.03 \%)$. The participants were unable to detect the RF status better than chance with $18.52 \%$ correctly identified trials. No participant was able to correctly identify all 3 exposure conditions.

\section{Cognitive Tasks}

The mean RTs and standard deviations for each cognitive task and the significance of each paired samples $t$ test are presented in Table I. As shown in Figure 3, there was significant decrease in RT during the exposure conditions compared to Sham, $t(35)=2.070, p=.046, r^{2}$ $=.109$. This effect, however, was not significant using non-interpolated data $(p=.052)$. No other RT effects were detected in either the visual discrimination tasks or the Sternberg working memory task when comparing Sham against the exposure conditions or the Low against the High RF condition, either during or following exposure.

The mean response sensitivity and standard deviations for each cognitive task and the significance of each paired samples $t$ test are presented in Table II. There were no significant differences in response sensitivity when comparing Sham against the exposure conditions or the Low against the High RF condition, either during or following exposure.

The mean response bias and standard deviations for each cognitive task and the significance of each paired samples $t$ test are presented in Table III. There were no significant differences in response bias when comparing Sham against the exposure conditions or the Low against the High RF condition, either during or following exposure. 
[Insert Tables I, Table II, Table III here]

[Insert Figure 3 here] 


\section{Discussion}

The results of the present study indicate that exposure to PM RF influences cognitive performance in the Sternberg working memory task. Specifically, a significant decrease in RT was revealed during the active exposure conditions compared to Sham. This suggests that exposure to PM RF may have a positive influence on cognitive performance. This effect, however, was not found to be dose dependent. While the exploratory analysis revealed that this effect was not significant using the non-interpolated data, the interpolation was used to preserve the sample size and counterbalancing. Therefore, because the non-interpolated data set contained fewer participants, the significance of this effect was expected to be reduced in the non-interpolated dataset.

While the present study's findings are consistent with early reports that exposure to PM RF affects working memory performance (Koivisto et al., 2000, Regel et al., 2007a, Regel et al., 2007b), the majority of the literature has not found such an influence (Haarala et al., 2003, Haarala et al., 2004, Haarala et al., 2007, Krause et al., 2007, Leung et al., 2011). Although we cannot be conclusive, a number of reasons related to the methodological improvements employed by this study may explain the results.

The working memory task used in the present study differs markedly from the N-back task, which is the task that has been typically utilised in provocation studies assessing the effect of PM RF exposure on working memory performance. It seems that the N-back task was used primarily because of its perceived face validity and (in latter studies) as a means to replicate previously reported effects. The problem, however, is that the N-back task is limited in its ability to control for individual differences in working memory performance, as well as learning, floor and ceiling effects. These confounds have the potential to add large amounts of noise to the data, thereby masking potentially real effects. The Sternberg working memory task overcomes these confounds by utilising a greater number of difficulty levels to calibrate 
the task to each individual's cognitive ability. This calibration, alongside sufficiently long practice blocks prior to each experimental session, increases sensitivity and thus the ability to detect any potential effect of PM RF exposure on working memory performance.

To date, only one other study has accounted for individual differences in working memory performance by tailoring cognitive task difficulty to participants' individual ability. After calibrating the N-back task to a level which was 'difficult, but achievable for each participant,' Leung et al. (2011) did not find any difference in accuracy or RT during PM RF exposure compared to Sham. However, in a 3G (W-CDMA) condition, Leung et al. (2011) found a significant decrease in accuracy in an adolescent group compared to Sham. The present study was able to control for a greater amount of variation in individual differences in performance, and thus remove the noise which may have masked any effects.

As the only known mechanism of interaction between RF and the human body is thermal (Adair and Black, 2003), it is possible that whole body thermoregulatory processes play an important role in mediating the changes in the brain's electrical activity and any potentially associated functional effects resulting from exposure to PM RF. To reduce thermally induced variability, the present study clamped skin temperature to a thermo-neutral state. As this is the first study to attempt to reduce the influence of this potential confound, this should be further explored in future studies.

Variation in exposure setups and SAR profiles and a lack of detailed dosimetric data has made it difficult to compare and replicate previously reported effects (Boutry et al., 2008, Regel and Achermann, 2011). While it is possible that certain brain regions need to be adequately exposed to PM RF to produce an effect, it should be noted that because the SAR distribution produced by the present planar exposure system is more homogenous than mobile phone hand-set exposure, this present exposure differs significantly to the more 
localised exposure produced by a typical mobile phone. Therefore, the results of this study can only reflect whether RF related bioeffects can occur at the maximum exposure level anywhere within the exposed hemisphere (Loughran et al., 2008). Thus, the present study cannot definitively comment on whether cognitive performance is influenced by PM RF emitted by mobile phones, nor can it comment on the effect of long term exposure or the effect of exposure on different age groups within the population. Indeed, the World Health Organisation has identified research investigating acute effects of PM RF exposure on cognition and EEG with children as a priority. While some studies have not found effects of PM RF exposure on cognitive performance in children and adolescents (Preece et al., 2005, Haarala et al., 2005, Leung et al., 2011), the methodology used in the present study may prove to be more sensitive, and should be considered in future research with children.

A number of factors may have limited this study. First, while the statistical analysis controlled for comparison-wise error by restricting the planned contrasts to degrees of freedom error (without multiple comparison adjustment) (Tabachnick and Fidell, 2013),this method does not control for experiment-wise error. Second, it is possible that the exposure levels were affected by the EEG electrodes. However, the potential for this to occur has been explored in great detail and is unlikely to have influenced the results. Generally, EEG electrode leads have been found to produce a shielding effect, reducing the SAR in head regions close to the antenna and also where the maximum value is obtained. The reduction in SAR tends to be less than 20\% (Murbach et al., 2014, Hamblin et al., 2007). These findings indicate that electrode configurations do not act like an antenna, and therefore do not enhance SAR. Furthermore, as the reductions have been found to be less than $20 \%$, electrode configurations are also not thought to greatly attenuate SAR to the point where results are influenced (Murbach et al., 2014, Hamblin et al., 2007). 
Whether small variations in performance on cognitive tasks as a result of exposure to PM RF constitute any meaningful effects in real life situations remains a valid question. In a critical review of this field, Regel and Achermann (2011) hypothesize that if such elementary motor reactions are influenced by exposure to PM RF, effects on higher cognitive functions may be even stronger. It is also possible, however, that the significant cognitive performance effects found in some RF-EMF provocation studies have occurred unpredictably and independent of task type (Regel and Achermann, 2011). This may explain why the significant differences found in the present study only occurred in one cognitive task on one variable. But while the results of the present study indicate that there is a slight change in performance, it is important that the methodology implemented in this study is replicated before determining whether such an effect is meaningful.

In conclusion, the present study has shown that PM RF exposure influences cognitive performance in a working memory task. While the majority of the literature has not found an effect of PM RF exposure on cognitive performance, it is possible that the methodological improvements employed in the present study increased sensitivity, and thus the ability to detect potential effects. However, as this is the first PM RF provocation study to implement these improvements, replication is required in order to determine whether these effects represent more than chance findings. 


\section{Acknowledgements}

The authors are thankful to Manuel Murbach (IT'IS Foundation, Zurich, Switzerland) for his technical support with the sXh920 planar exposure system.

\section{Disclosure Statement}

RM is employed by the Australian Mobile Telecommunications Association. The authors report no other conflicts of interest. The authors alone are responsible for the content and writing of the paper.

\section{Funding}

This study was funded by the National Health and Medical Research Council of Australia (Grant 1042464) and the Electric Power Research Institute (Grant 00-10003301). 


\section{References}

Adair, E. R. \& Black, D. R. 2003. Thermoregulatory responses to RF energy absorption. Bioelectromagnetics, 24, S17-S38.

Barth, A., Ponocny, I., Gnambs, T. \& Winker, R. 2012. No effects of short-term exposure to mobile phone electromagnetic fields on human cognitive performance: A meta-analysis. Bioelectromagnetics, 33, 159-165.

Boutry, C. M., Kuehn, S., Achermann, P., Romann, A., Keshvari, J. \& Kuster, N. 2008. Dosimetric evaluation and comparison of different RF exposure apparatuses used in human volunteer studies. Bioelectromagnetics, 29, 11-19.

Croft, R. J., Chandler, J. S., Barry, R. J., Cooper, N. R. \& Clarke, A. R. 2005. EOG correction: a comparison of four methods. Psychophysiology, 42, 16-24.

Croft, R. J., Hamblin, D. L., Spong, J., Wood, A. W., Mckenzie, R. J. \& Stough, C. 2008. The effect of mobile phone electromagnetic fields on the alpha rhythm of human electroencephalogram. Bioelectromagnetics, 29, 1-10.

Croft, R. J., Leung, S., Mckenzie, R. J., Loughran, S. P., Iskra, S., Hamblin, D. L. \& Cooper, N. R. 2010. Effects of $2 \mathrm{G}$ and $3 \mathrm{G}$ mobile phones on human alpha rhythms: Resting EEG in adolescents, young adults, and the elderly. Bioelectromagnetics, 31, 434-444.

Curcio, G., Ferrara, M., Moroni, F., D'inzeo, G., Bertini, M. \& De Gennaro, L. 2005. Is the brain influenced by a phone call? An EEG study of resting wakefulness. Neuroscience Research, 53, 265-270.

Haarala, C., Bergman, M., Laine, M., Revonsuo, A., Koivisto, M. \& Hämäläinen, H. 2005. Electromagnetic field emitted by $902 \mathrm{MHz}$ mobile phones shows no effects on children's cognitive function. Bioelectromagnetics, 26, S144-S150.

Haarala, C., Björnberg, L., Ek, M., Laine, M., Revonsuo, A., Koivisto, M. \& Hämäläinen, H. 2003. Effect of a $902 \mathrm{MHz}$ electromagnetic field emitted by mobile phones on human cognitive function: A replication study. Bioelectromagnetics, 24, 283-288.

Haarala, C., Ek, M., Bjornberg, L., Laine, M., Revonsuo, A., Koivisto, M. \& Hamalainen, H. 2004. $902 \mathrm{MHz}$ mobile phone does not affect short term memory in humans. Bioelectromagnetics, 25, 452 - 456.

Haarala, C., Takio, F., Rintee, T., Laine, M., Koivisto, M., Revonsuo, A. \& Hamalainen, H. 2007. Pulsed and continuous wave mobile phone exposure over left versus right hemisphere: Effects on human cognitive function. Bioelectromagnetics, 28, 289 - 295.

Hamblin, D. L., Anderson, V., Mcintosh, R. L., Mckenzie, R. J., Wood, A. W., Iskra, S. \& Croft, R. J. 2007. EEG Electrode Caps Can Reduce SAR Induced in the Head by GSM900 Mobile Phones. IEEE Transactions on Biomedical Engineering, 54, 914-920.

Health Canada 2015. Safety code 6: Limits of human exposure to radiofrequency electromagnetic energy in the frequency range from $3 \mathrm{kHz}$ to $300 \mathrm{GHz}$. Canada: Consumer and Clinical Radiation Protection Bureau.

Health Council of the Netherlands 2009. Electromagnetic fields: Annual update 2008. The Hague: Health Council of the Netherlands.

Huber, R., Achermann, P., Graf, T., Cote, K. A., Wittmann, L., Gallmann, E., Matter, D., Schuderer, J., Kuster, N. \& Borbély, A. A. 2000. Exposure to pulsed high-frequency electromagnetic field during waking affects human sleep EEG. NeuroReport, 11, 3321-3325.

Huber, R., Buck, A., Achermann, P., Treyer, V., Borbély, A. A., Schuderer, J., Gottselig, J. M., Landolt, H. P., Werth, E., Berthold, T. \& Kuster, N. 2002. Electromagnetic fields, such as those from mobile phones, alter regional cerebral blood flow and sleep and waking EEG. Journal of Sleep Research, 11, 289-295.

Huber, R., Treyer, V., Schuderer, J., Berthold, T., Buck, A., Kuster, N., Landolt, H. P. \& Achermann, P. 2005. Exposure to pulse-modulated radio frequency electromagnetic fields affects regional cerebral blood flow. The European Journal of Neuroscience, 21, 1000-1006.

Koivisto, M., Krause, C. M., Revonsuo, A., Laine, M. \& Hämäläinen, H. 2000. The effects of electromagnetic field emitted by GSM phones on working memory. Neuroreport, 11, 16411643. 
Krause, C., Pesonen, M., Bjornberg, C. \& Hamalainen, H. 2007. Effects of pulsed and continuous wave $902 \mathrm{MHz}$ mobile phone exposure on brain oscillatory activity during cognitive processing. Bioelectromagnetics, 28, 296 - 308.

Leung, S., Hamblin, D., Simpson, D., Croft, R. J., Mckenzie, R. J., Iskra, S., Silber, B., Cooper, N. R., O'neill, B., Cropley, V. \& Diaz-Trujillo, A. 2011. Effects of 2G and 3G mobile phones on performance and electrophysiology in adolescents, young adults and older adults. Clinical Neurophysiology, 122, 2203-2216.

Loughran, S. P., Benz, D. C., Schmid, M. R., Murbach, M., Kuster, N. \& Achermann, P. 2013. No increased sensitivity in brain activity of adolescents exposed to mobile phone-like emissions. Clinical Neurophysiology, 124, 1303-1308.

Loughran, S. P., Mckenzie, R. J., Anderson, V., Mcintosh, R. L. \& Croft, R. J. 2008. Dosimetric evaluation and comparison of different RF exposure apparatuses used in human volunteer studies. Bioelectromagnetics, 29, 242-243.

Loughran, S. P., Wood, A. W., Barton, J. M., Croft, R. J., Thompson, B. \& Stough, C. 2005. The effect of electromagnetic fields emitted by mobile phones on human sleep. Neuroreport, 16, 1973-6.

Murbach, M., Christopoulou, M., Crespo-Valero, P., Achermann, P. \& Kuster, N. 2012. Exposure system to study hypotheses of ELF and RF electromagnetic field interactions of mobile phones with the central nervous system. Bioelectromagnetics, 33, 527-533.

Murbach, M., Neufeld, E., Christopoulou, M., Achermann, P. \& Kuster, N. 2014. Modeling of EEG electrode artifacts and thermal ripples in human radiofrequency exposure studies. Bioelectromagnetics, 35, 273-83.

Perentos, N., Croft, R. J., Mckenzie, R. J., Cvetkovic, D. \& Cosic, I. 2007. Comparison of the effects of continuous and pulsed mobile phone like RF exposure on the human EEG. Australasian Physics \& Engineering Sciences in Medicine, 30, 274-280.

Preece, A. W., Goodfellow, S., Wright, M. G., Butler, S. R., Dunn, E. J., Johnson, Y., Manktelow, T. C. \& Wesnes, K. 2005. Effect of $902 \mathrm{MHz}$ mobile phone transmission on cognitive function in children. Bioelectromagnetics, 26, S138-S143.

Regel, S. J. \& Achermann, P. 2011. Cognitive performance measures in bioelectromagnetics research: Critical evaluation and recommendations. Environmental Health, 10, 10-10.

Regel, S. J., Gottselig, J. M., Schuderer, J., Tinguely, G., Retey, J. V., Kuster, N., Landolt, H. P. \& Achermann, P. 2007a. Pulsed radio frequency radiation affects cognitive performance and the waking electroencephalogram. Neuroreport, 18, 803-7.

Regel, S. J., Tinguely, G., Schuderer, J., Adam, M., Kuster, N., Landolt, H. P. \& Achermann, P. 2007b. Pulsed radio-frequency electromagnetic fields: Dose-dependent effects on sleep, the sleep EEG and cognitive performance. Journal of Sleep Research, 16, 253-258.

Scenihr 2009. SCENIHR (Scientific Committee on Emerging and Newly Identified Health Risks). Health Effects of Exposure to EMF. Brussels: European Commission Directorate-General for Health and Consumers.

Schmid, M. R., Loughran, S. P., Regel, S. J., Murbach, M., Bratic Grunauer, A., Rusterholz, T., Bersagliere, A., Kuster, N. \& Achermann, P. 2012. Sleep EEG alterations: Effects of different pulse-modulated radio frequency electromagnetic fields. J Sleep Res, 21, 50-8.

Stanislaw, H. \& Todorov, N. 1999. Calculation of signal detection theory measures. Behaviour Research Methods, Instruments and Computers, 31, 137-149.

Sternberg, S. 1966. High-speed scanning in human memory. Science. American Association for the Advancement of Science.

Tabachnick, B. G. \& Fidell, L. S. 2013. Using multivariate statistics, Boston, Pearson Education.

Valentini, E., Ferrara, M., Presaghi, F., De Gennaro, L. \& Curcio, G. 2010. Republished review: Systematic review and meta-analysis of psychomotor effects of mobile phone electromagnetic fields. Postgraduate Medical Journal, 67, 708 - 716.

World Health Organisation. 2014. World Health Organisation Fact Sheet No. 193: Electromagnetic Fields and Public Health: Mobile Phones [Online]. World Health Organisation Available: http://www.who.int/mediacentre/factsheets/fs193/en/index.html [Accessed 05/12/2015. 
Table I: Mean reaction times (ms) and standard deviations for each cognitive task in each exposure condition with $p$ values for Sham vs Exposure and Low vs High comparisons (values significant at $p<.05$ are in bold).

\begin{tabular}{|c|c|c|c|c|c|c|}
\hline \multirow[t]{2}{*}{ Condition } & \multicolumn{2}{|c|}{ Perceptual Easy } & \multicolumn{2}{|c|}{ Perceptual Difficult } & \multicolumn{2}{|c|}{ Sternberg } \\
\hline & $\begin{array}{c}\text { BL1 } \\
\text { M (SD) }\end{array}$ & $\begin{array}{c}\text { BL2 } \\
\text { M (SD) }\end{array}$ & $\begin{array}{c}\text { BL1 } \\
\text { M (SD) }\end{array}$ & $\begin{array}{c}\text { BL2 } \\
\mathrm{M}(\mathrm{SD})\end{array}$ & $\begin{array}{c}\text { BL1 } \\
\mathrm{M}(\mathrm{SD})\end{array}$ & $\begin{array}{c}\text { BL2 } \\
\text { M (SD) }\end{array}$ \\
\hline Sham & $358(42)$ & $359(45)$ & $368(39)$ & $362(63)$ & 877 (136) & $367(148)$ \\
\hline Low RF & $360(42)$ & $350(37)$ & $377(63)$ & $364(45)$ & 833 (138) & $872(140)$ \\
\hline High RF & $359(42)$ & $356(37)$ & $374(42)$ & $367(36)$ & $833(130)$ & $873(138)$ \\
\hline \multicolumn{7}{|l|}{$p$ values } \\
\hline Sham vs Exposure & .762 & .207 & .192 & .330 & .045 & .845 \\
\hline Low vs High & .850 & .162 & .870 & .436 & .989 & .983 \\
\hline
\end{tabular}


Table II: Mean response sensitivity (Grier's A') and standard deviations for each cognitive task in each exposure condition with $p$ values for Sham vs Exposure and Low vs High comparisons.

\begin{tabular}{|c|c|c|c|c|c|c|}
\hline \multirow[t]{2}{*}{ Condition } & \multicolumn{2}{|c|}{ Perceptual Easy } & \multicolumn{2}{|c|}{ Perceptual Difficult } & \multicolumn{2}{|c|}{ Sternberg } \\
\hline & $\begin{array}{c}\text { BL1 } \\
\mathrm{M}(\mathrm{SD})\end{array}$ & $\begin{array}{c}\text { BL2 } \\
\text { M (SD) }\end{array}$ & $\begin{array}{c}\text { BL1 } \\
\text { M (SD) }\end{array}$ & $\begin{array}{c}\text { BL2 } \\
\text { M (SD) }\end{array}$ & $\begin{array}{c}\text { BL1 } \\
\text { M (SD) }\end{array}$ & $\begin{array}{c}\text { BL2 } \\
\mathrm{M}(\mathrm{SD})\end{array}$ \\
\hline Sham & $.94(.04)$ & $.94(.05)$ & $.91(.09)$ & $.90(.08)$ & $.85(.10)$ & $.86(.07)$ \\
\hline Low RF & $.94(.06)$ & $.94(.06)$ & $.91(.08)$ & $.91(.08)$ & $.85(.08)$ & $.85(.09)$ \\
\hline High RF & $.93(.06)$ & $.94(.05)$ & $.92(.07)$ & $.91(.08)$ & $.86(.07)$ & $.86(.07)$ \\
\hline \multicolumn{7}{|l|}{$p$ values } \\
\hline Sham vs Exposure & .454 & .741 & .528 & .078 & .758 & .515 \\
\hline Low vs High & .391 & .920 & .297 & .905 & .512 & .228 \\
\hline
\end{tabular}


Table III: Mean response bias (Grier's B') and standard deviations for each cognitive task in each exposure condition with $p$ values for Sham vs Exposure and Low vs High comparisons.

\begin{tabular}{lcccccc}
\hline Condition & \multicolumn{3}{c}{ Perceptual Easy } & \multicolumn{2}{c}{ Perceptual Difficult } & \multicolumn{2}{c}{ Sternberg } \\
\cline { 2 - 7 } & BL1 & BL2 & BL1 & BL2 & BL1 & BL2 \\
& M (SD) & M (SD) & M (SD) & M (SD) & M (SD) & M (SD) \\
\hline Sham & $.10(50)$ & $.08(.52)$ & $.25(.45)$ & $.23(.46)$ & $.28(.36)$ & $.29(.28)$ \\
Low RF & $.14(.48)$ & $.07(.52)$ & $.30(.41)$ & $.28(.45)$ & $.25(.33)$ & $.25(.29)$ \\
High RF & $.11(.50)$ & $.08(.41)$ & $.22(.32)$ & $.21(.43)$ & $.28(.32)$ & $.30(.30)$ \\
\hline
\end{tabular}

$p$ values

$\begin{array}{lllllll}\text { Sham vs Exposure } & .583 & .964 & .882 & .842 & .863 & .636 \\ \text { Low vs High } & .607 & .907 & .197 & .134 & .512 & .228\end{array}$

a)
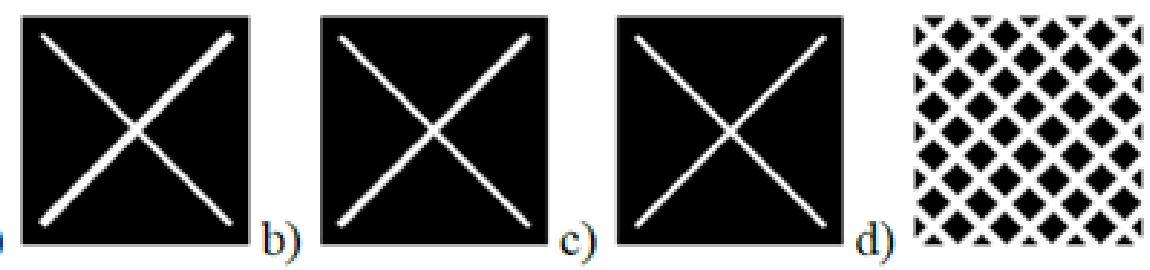

Figure 1: Examples of the stimuli used in the visual discrimination task. (a) Is an example of an easy target stimulus, (b) is an example of a difficult target stimulus (c) is a non-target stimulus and (d) is the mask used between stimulus presentations. 


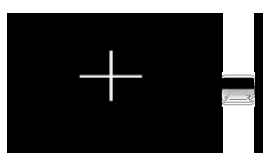

Fixation

Cross

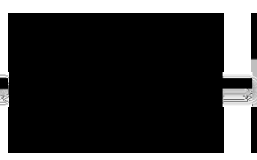

Pause

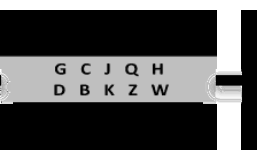

Memory Set

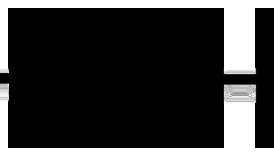

Retention

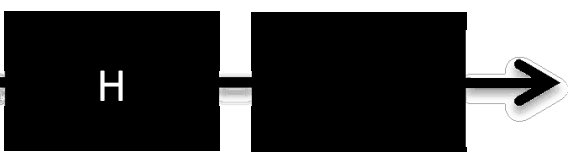

Probe \& Pause Response

Figure 2: The modified Sternberg working memory task design and progression. A fixation cross (800 ms) was followed by a $1000 \mathrm{~ms}$ pause. The memory set was then presented (4000 ms) and was followed by a blank screen retention period (3000 ms). Following this, a probe stimulus appeared $(2000 \mathrm{~ms})$ during which time participants had to respond with a button press if the probe was present in the preceding memory set, or not respond if the probe was not present in the preceding memory set. A blank screen pause (2000 ms) concluded the trial before the onset of the next trial.

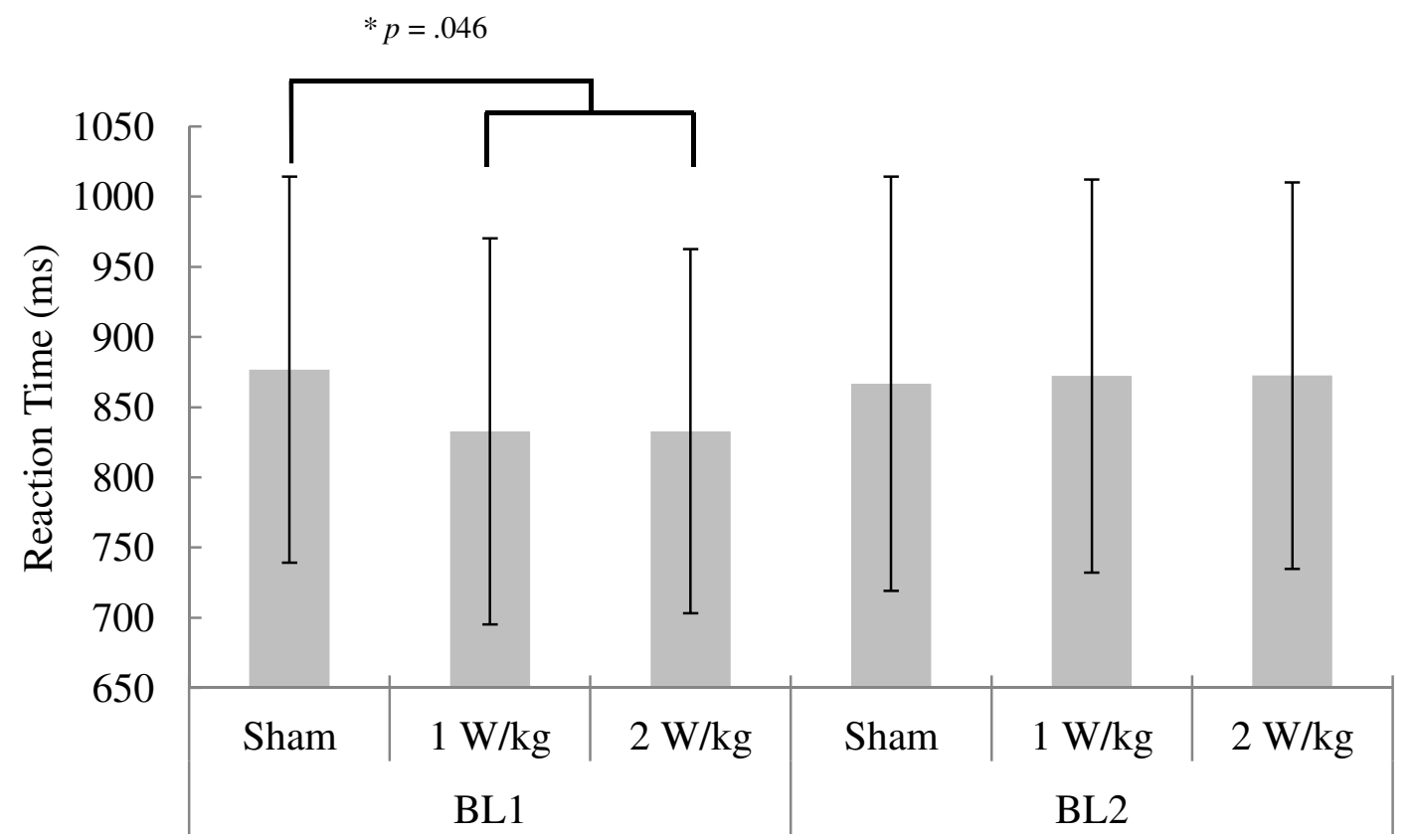

Figure 3: Mean reaction times (ms) in the Sternberg working memory task as a function of condition and block (BL1: during exposure, BL2: post exposure). Paired samples $t$ tests showed a significant decrease in reaction time during exposure when compared to sham. Error bars represent standard deviation. 